\title{
EL EFECTO DEL INSTITUTO NACIONAL: EVIDENCIA A PARTIR DE UN DISEÑO DE REGRESIÓN DISCONTINUA*
}

\author{
Alonso Bucarey \\ MIT \\ Pablo Muñoz \\ Universidad de Chile
}

\author{
Miguel Jorquera \\ Fiscalía Nacional Económica \\ Sergio Urzúa \\ Universidad de Maryland
}

Resumen: Este trabajo presenta evidencia del impacto causal
de haber asistido a un liceo emblemático sobre la Prueba de
Selección Universitaria (PSU). En particular, analizamos
el caso del Instituto Nacional. Nuestra estrategia empírica

Alonso Bucarey. Ingeniero Comercial y Magíster Economía de la Universidad de Chile. Actualmente es estudiante del doctorado en Economía de MIT. bucarey@mit.edu.

Miguel JoRquera. Ingeniero Comercial y trabaja en la Fiscalía Nacional Económica.mjorquera@fne.gob.cl.

PaBlo MuÑoz. Ingeniero Comercial y Magíster en Análisis Económico de la Universidad de Chile.pabmunoz@fen.uchile.cl.

Sergio Urzúa. PhD en Economía de la Universidad de Chicago y profesor de la Universidad de Maryland. urzua@econ.umd.edu.

* Agradecemos al Instituto Nacional por brindarnos acceso a sus archivos. Toda la información se utilizó de tal forma de salvaguardar la identidad de las personas. El documento no presenta ninguna información que permita identificar a individuos. Se agradecen los comentarios de Patricia Acosta, Luis Elmes, Arturo Fontaine y dos anónimos referees. 
se basa en la utilización de una regresión discontinua (RD). Específicamente, empleando datos de la prueba de selección de este establecimiento, comparamos los resultados en PSU de jóvenes que son similares al momento de postular al establecimiento, pero que difieren en cuanto a haber asistido o no al Instituto Nacional. Los resultados sugieren que estudiar en el Instituto Nacional tiene un efecto de 26,13 puntos $(0,25$ desviaciones estándar) adicionales en el promedio de PSU. Interpretamos este resultado como un efecto causal local, es decir, un efecto en la vecindad de la discontinuidad generada por el puntaje de corte utilizado en el proceso de selección de alumnos.

Palabras clave: Liceos de excelencia, regresiones discontinuas, impacto de educación.

Recibido: septiembre 2013; aceptado: enero 2014.

\section{THE EFFECT OF ATTENDING AN ELITE PUBLIC HIGH-SCHOOL IN CHILE. THE CASE OF INSTITUTO NACIONAL}

Abstract: This paper estimates the effect of attending one of the most important elite public high schools in Chile, the Instituto Nacional. Using data from its admission tests, we implement a regression discontinuity approach to estimate the impact of this high school on students' academic performance. Our measure of academic impact is the Chilean college admission exam (PSU). The estimated impact associated with the event "attending the Instituto Nacional" is 26,13 additional points on PSU (0.25 standard deviations). Due to its nature, we interpret this result as a local causal effect.

Keywords: Elite schools, regression discontinuity, impact of education.

Received: September 2013; accepted: January 2014.

\section{Introducción}

E valor agregado de los liceos de excelencia en Chile ha sido un tema reciente en el debate público. Se ha argumentado que el buen resultado de estos establecimientos, tanto en las pruebas del Sistema de Medición de la Calidad de la Educación (SIMCE) como de admisión universitaria (PSU), se explicaría por el proceso de selección que éstos realizan y no por su capacidad para generar valor agregado. Este 
estudio contribuye a esta discusión al presentar evidencia causal del impacto de asistir al Instituto Nacional, probablemente el establecimiento educacional público más conocido entre los liceos emblemáticos y un modelo a seguir por los establecimientos de excelencia.

Nuestro análisis empírico utiliza datos administrativos del proceso de admisión de este establecimiento educacional, combinado con los resultados de sus alumnos en la prueba de Selección Universitaria (PSU). Esto nos permite utilizar una estrategia de identificación que determina el efecto causal de asistir a este establecimiento. En particular, implementamos un diseño de regresión discontinua, el que ha sido utilizado anteriormente en la literatura. Esta metodología se basa en la comparación de los resultados en la PSU de estudiantes que estuvieron por arriba (matriculados) y por debajo (no matriculados) del puntaje de corte en la prueba de admisión del Instituto Nacional.

La evidencia internacional ha entregado conclusiones disímiles sobre el efecto de este tipo de liceos en los resultados de los alumnos. Por una parte, en países desarrollados como Estados Unidos e Inglaterra los resultados sugieren que este tipo de liceos no tiene impacto académico significativo, típicamente medido por los resultados en las pruebas de selección universitaria de sus estudiantes. Esto implica que si los alumnos de estos liceos hubieran asistido a su segunda mejor alternativa habrían obtenido un resultado similar en las respectivas pruebas de selección universitaria. Por otra parte, los estudios sobre este tipo de establecimientos en países en desarrollo, como Rumania y Trinidad y Tobago, sugieren que la asistencia a estos liceos tendría un impacto positivo en el puntaje de admisión a las universidades y en el puntaje de otras pruebas al finalizar los estudios secundarios. Nuestros resultados son consistentes con la evidencia de estos últimos países.

Ahora bien, existen múltiples razones que pueden explicar la ambigüedad en los resultados. Por una parte, distintos estudios han planteado canales que explicarían por qué los alumnos pueden beneficiarse de la asistencia a un liceo de excelencia (Dobbie y Fryer, 2011). En primer lugar, los efectos pares. La literatura ha mostrado la importancia de las interacciones sociales y de las redes como un elemento de formación de habilidades, de desarrollo de capital humano y capital social (Sacerdote, 2011). La asistencia a establecimientos emblemáticos puede proveer este tipo de beneficios a los estudiantes. En segundo lugar, dado el mayor nivel académico al interior de los cursos, la labor del docente se potencia al enfocar sus esfuerzos en alumnos con un 
desempeño más homogéneo (sobre la mediana), mejorando entonces su trabajo como educador (Duflo, Dupas y Kremer, 2011). Por otra parte, la literatura también ha levantado dudas respecto del real impacto de estos establecimientos. Pop-Eleches y Urquiola (2011), por ejemplo, han planteado que los alumnos podrían verse perjudicados al estudiar en un liceo de excelencia, puesto que la asistencia a ellos podría sustituir otro tipo de inversiones en educación que antes realizaba la familia. Nuestra metodología no permite distinguir los efectos positivos o negativos, entregando el efecto neto para el caso del Instituto Nacional.

Este tema es altamente relevante en el caso de Chile, pues desde 2011 el número de liceos de excelencia se ha incrementado de 25 a 60. Este crecimiento ha sido el resultado de una política pública que ha pretendido, a partir de la matrícula en estos establecimientos, otorgar una vía de movilidad social para estudiantes de escasos recursos ${ }^{1}$. Estos colegios además han tenido como modelo a los liceos de excelencia tradicionales, los que entre otras características tienen una alta demanda, seleccionan a sus alumnos mediante pruebas de conocimientos y habilidades, y tienen excelentes resultados en distintas medidas de desempeño académico.

El presente trabajo se estructura como sigue: en la sección 2 se presenta una revisión de la literatura y en la sección 3 se detallan algunos antecedentes sobre el Instituto Nacional. La sección 4 describe los datos utilizados en este trabajo, mientras que la sección 5 describe la metodología de regresión discontinua que utilizamos. Posteriormente, en la sección 6 se presentan los resultados obtenidos y los distintos ejercicios de robustez. La sección 7 concluye.

\section{Revisión de la literatura}

Una característica distintiva de los colegios de excelencia en el mundo es que sus alumnos son seleccionados en base a sus méritos académicos. A continuación detallamos los estudios más relevantes que han analizado la efectividad de este tipo de establecimientos sobre los resultados académicos de los estudiantes.

${ }^{1}$ Para obtener un mayor detalle sobre los liceos bicentenarios, proceda a revisar la información brindada por el Ministerio de Educación en su página web: http://www.liceosbicentenario.mineduc.cl/index.php?id_portal=57 
Abdulkadiroğlu, Angrist y Pathak (2012) analizan el caso de los Estados Unidos. Utilizando datos de puntaje de selección de colegios de excelencia en Boston y Nueva York, estos autores implementan una estrategia de identificación basada en una metodología de regresión discontinua, similar a la utilizada en nuestro estudio. Sus resultados muestran que asistir a un liceo de excelencia no tiene efecto sobre el puntaje MCAS (similar a la evaluación SIMCE en Chile) ni en PSAT (similar a la PSU para el caso de Chile). Estos resultados son consistentes con los discutidos en Dobbie y Fryer (2011), quienes también examinan datos de Nueva York en base a una estrategia de identificación similar a la utilizada por Abdulkadiroğlu, Angrist y Pathak (2012). Un punto relevante planteado en este estudio es que dado que quienes postulan a liceos de excelencia suelen tener como alternativa colegios de buena calidad e incluso otros liceos de excelencia, lo que se evalúa en dicho caso es el efecto de asistir a un mejor colegio y no el efecto de "liceos de excelencia".

Para Inglaterra, Clark (2008) analiza el efecto de asistir a establecimientos que seleccionan en base a criterio académico. En este caso, los resultados sugieren que no existe un efecto en las pruebas rendidas al finalizar la educación secundaria, aunque sí se observan resultados positivos y significativos en la probabilidad de acceder a la universidad.

Existe una serie de estudios que utilizan los sorteos que algunos distritos en los Estados Unidos realizan para asignar cupos en liceos que tienen excesiva demanda. Éstos no necesariamente son de excelencia, pero sí de alta demanda. Cullen et al. (2006) encuentran que los estudiantes seleccionados para asistir a mejores escuelas en Chicago con este mecanismo no muestran mejoras en los puntajes de los tests. Por otro lado, Hastings y Weinstein (2008) encuentran un efecto positivo y significativo en resultados de tests cognitivos posteriores a la asistencia a una mejor escuela entre los estudiantes seleccionados por el sorteo en Charlotte-Mecklonburg al ser comparados con quienes no fueron seleccionados.

El tema de los establecimientos de excelencia y el impacto de la selección también ha sido abordado en países en vías de desarrollo, aunque con resultados cualitativamente diferentes a los antes mencionados. Pop-Eleches y Urquiola (2011) realizan un estudio similar a los llevados a cabo en los Estados Unidos, pero para el caso de Rumania. Este estudio reúne casi dos mil regresiones discontinuas para dicho 
país para diferentes liceos. Sus resultados muestran que la asistencia a una mejor escuela tiene un efecto de hasta 0,1 desviaciones estándar en puntaje de admisión a las universidades. Adicionalmente, este trabajo investiga el efecto de comportamiento que tiene sobre los padres la asistencia a una mejor escuela. La evidencia sugiere que, si bien las mejores escuelas tienen padres más involucrados, a nivel individual no hay efectos positivos en el comportamiento de los padres en términos de esfuerzo e inversión. De hecho, las estimaciones muestran un efecto negativo de asistir a este tipo de establecimientos en el tiempo que dedican los padres a ayudar a sus hijos en las labores escolares (tareas). En este contexto, Pop-Eleches y Urquiola (2011) plantean que asistir a una mejor escuela es hasta cierto punto un sustituto de la inversión en tiempo de los padres. Adicionalmente, a nivel de estudiantes encuentran que la asistencia a una mejor escuela aumenta el tiempo que los estudiantes dedican a realizar sus tareas.

En la misma línea, Jackson (2010) encuentra un efecto positivo e importante en las pruebas realizadas al finalizar la educación secundaria en Trinidad y Tobago. Este estudio utiliza la discontinuidad generada por el puntaje de ingreso como variable instrumental y también como un diseño de regresión discontinua. Zhang (2010), por otra parte, evalúa el efecto de las Magnet $S_{\text {chools }}^{2}$ para una zona de China en donde la matrícula a este tipo de establecimientos es otorgada en base a una lotería. El autor encuentra, al igual que en los estudios para Estados Unidos, que no existe un efecto significativo de la asistencia a este tipo de escuelas sobre resultados en pruebas cognitivas posteriores. En este contexto, el estudio discute las dificultades de explicar racionalmente el esfuerzo de los padres para que sus hijos sean seleccionados en este tipo de escuelas, proponiendo como explicaciones: i) que para los padres es imposible distinguir el resultado promedio de estas escuelas con el valor agregado que entregan, o ii) que para los investigadores es imposible identificar otros beneficios inicialmente no observables que pueden derivarse de estas escuelas, como el acceso a redes de contactos.

Para Chile la evidencia es casi inexistente. Una excepción es el estudio de Valenzuela y Allende (2012). Estos autores utilizan un panel

${ }^{2}$ En la provincia de Wuhan estas escuelas cobran un arancel anual de USD 400 y ofrecen beca a estudiantes en base a mérito académico. Si bien no son directamente comparables con los liceos de excelencia, también muestran el efecto de asistir a una mejor escuela en términos generales. 
con pruebas estandarizadas (SIMCE) para alumnos en cuarto básico y segundo medio. Esto les permite comparar los resultados en SIMCE de estudiantes que en segundo medio se encontraban matriculados en alguno de los 30 liceos de excelencia considerados en el estudio (grupo de tratamiento), con aquellos de jóvenes que no fueron matriculados en este tipo de establecimientos (controles), pero que en cuarto básico tenían características similares a los tratados. La estrategia empírica utiliza estimadores de matching, encontrando que luego de controlar por efecto par y por varios observables, los liceos públicos de excelencia no tienen efecto alguno sobre el rendimiento en segundo medio. Una desventaja de este estudio es que las estimaciones pueden ser interpretadas de manera causal sólo en el caso en que los alumnos que postulan a liceos de excelencia no difieren en características no observables de quienes no lo hacen. Nuestro estudio se hace cargo de este posible problema utilizando la estrategia de regresión discontinua.

\section{Antecedentes}

El Instituto Nacional General José Miguel Carrera fue fundado el 10 de agosto de 1813 y es el más antiguo de los denominados liceos públicos de excelencia. El establecimiento destaca por tener el mejor rendimiento entre los colegios con dependencia educacional de tipo municipal (público) en las pruebas estandarizadas a nivel país (SIMCE y PSU). Esto le permite atraer estudiantes de toda la Región Metropolitana. La figura $n^{\circ} 1$ presenta la residencia de los alumnos matriculados en el establecimiento el año $2000^{3}$.

Se observa que los estudiantes del Instituto Nacional provienen de casi todas las comunas de la Región Metropolitana, aunque con una fuerte concentración en las zonas ubicadas al sur del establecimiento. Específicamente, las comunas que concentran el mayor número de postulantes seleccionados en el año 2000 y 2002 son Maipú, La Florida, Santiago, Puente Alto y Pudahuel. En estas cinco comunas habita el $42 \%$ del total de estudiantes seleccionados estos años. Esto permite aproximar una caracterización socioeconómica de los alumnos y mostrar los costos en que incurren las familias al decidir enviar a sus hijos

${ }^{3}$ Este año también fue seleccionado un estudiante de la comuna de Antofagasta. 
FIGURA $N^{\circ} 1: \quad$ RESIDENCIA DE LOS ESTUDIANTES SELECCIONADOS POR EL INSTITUTO NACIONAL, AÑO 2000

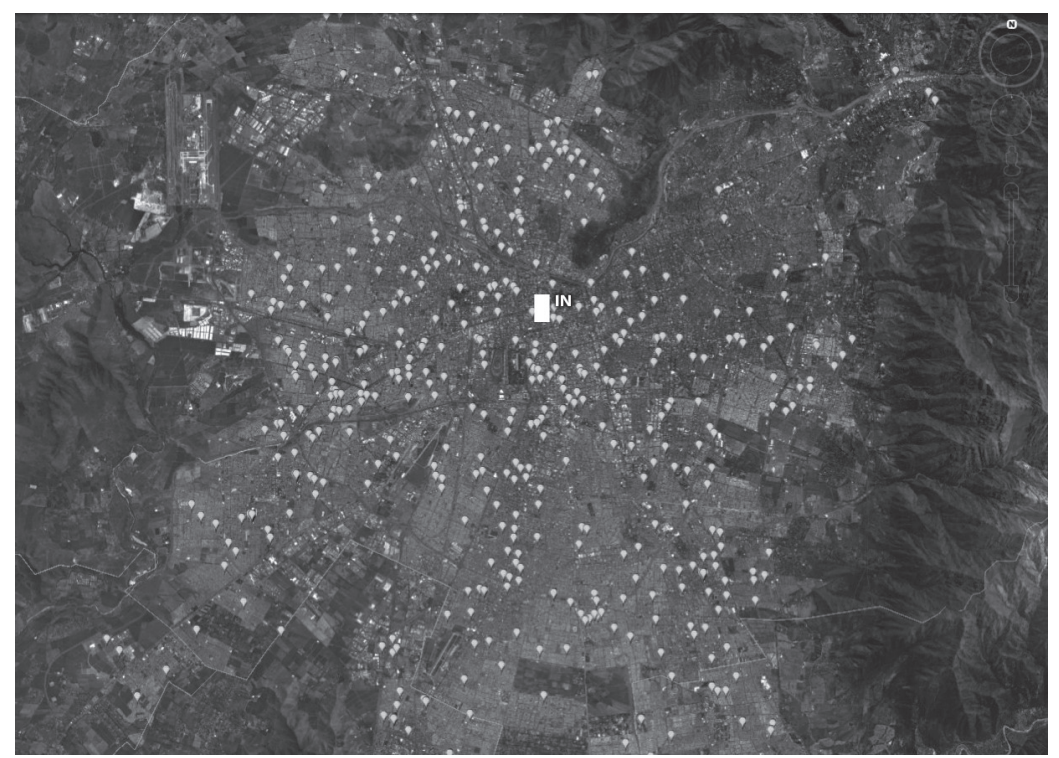

Fuente: Construcción propia en base a datos administrativos del Instituto Nacional.

al establecimiento. En particular, de acuerdo a datos de la Encuesta de Caracterización Socioeconómica Nacional de 2011 (CASEN 2011), el ingreso per cápita promedio en Maipú es \$260.868, \$262.839 en La Florida, \$455.573 en Santiago, \$165.795 en Puente Alto y \$157.942 en Pudahuel, mientras que el promedio simple de todas las comunas donde existe al menos un postulante es \$265.292. Además, con información del Programa de Naciones Unidas para el Desarrollo (PNUD 2012) es posible obtener la distancia promedio entre las comunas de Maipú, La Florida, Santiago, Puente Alto y Pudahuel respecto del Instituto Nacional. Estas distancias corresponden a $15 \mathrm{~km}, 20 \mathrm{~km}, 2 \mathrm{~km}, 25 \mathrm{~km}$ y 23 $\mathrm{km}$, respectivamente. Es decir, estudiar en el Instituto Nacional generalmente se asocia a altos tiempos de viaje del establecimiento al hogar y viceversa.

Respecto de su programa académico, el Instituto Nacional aplica un currículo educacional común con el resto de los establecimientos del 
país. Éste se fundamenta en las directrices dispuestas por el Ministerio de Educación, las que contemplan una formación científico-humanista junto al desarrollo de idiomas, actividades artísticas y deportivas. Este liceo cuenta con 6 niveles, los que abarcan desde $7^{\circ}$ básico hasta $4^{\circ}$ medio, y es posible ingresar al colegio por vía regular solo en el primero de estos niveles.

La postulación al establecimiento es un proceso que comienza en el mes de julio con la publicación del calendario y los requisitos del proceso. Éstos son: ingresar los antecedentes personales al sistema de postulación del establecimiento dentro de las semanas dispuestas, cancelar el arancel de admisión de $\$ 3.500$ (valor para el año 2013), presentar la documentación física acerca de los antecedentes personales y académicos junto a los antecedentes del apoderado 4 y rendir la prueba de admisión que se divide en preguntas en los tópicos de lenguaje y matemáticas. Si bien dicha prueba se basa en los contenidos correspondientes a los niveles de $5^{\circ}$ y $6^{\circ}$ básico, ésta también incluye preguntas enfocadas a medir habilidades cognitivas. Los resultados de la postulación se informan en el mes de noviembre. Finalmente, el proceso finaliza en diciembre con reuniones informativas para los apoderados de los alumnos seleccionados.

Respecto de la selección de los alumnos, ésta se basa en criterios académicos, donde el establecimiento realiza un ranking de los postulantes de acuerdo con un promedio ponderado entre sus calificaciones (promedio de notas en los niveles de $5^{\circ}$ básico y $6^{\circ}$ básico) y los resultados en la prueba de admisión. El primer ítem tiene asociado un porcentaje de $60 \%$ dentro del puntaje de postulación, mientras que el segundo se lleva el restante $40 \%$. Luego, el establecimiento establece un puntaje de corte de acuerdo con el número de vacantes disponibles. Los alumnos que tienen un puntaje mayor o igual a este corte son seleccionados, los alumnos con puntajes cercanos pero bajo éste son informados que se encuentran en lista de espera y el resto de los postulantes son inmediatamente descartados. Los alumnos no seleccionados continúan sus estudios en diferentes establecimientos aunque con fuerte presencia de algunos liceos de excelencia. A modo de ejemplo, el año

${ }^{4}$ Los padres ex alumnos del establecimiento pueden presentar documentación con respecto a su egreso del Instituto Nacional dentro del proceso de postulación de sus hijos. 
2002 el establecimiento recibió 3.800 postulaciones, de las cuales 3.125 no recibieron matrícula en el IN. Estos estudiantes se matricularon en 677 colegios, dentro de los cuales destacan cuatro por concentrar el $20 \%$ de los postulantes que no ingresan. En orden decreciente respecto del número de postulantes al IN, estos establecimientos son: Liceo Nacional de Maipú con 205 postulantes, Liceo de Aplicación con 186 postulantes, Internado Nacional Barros Arana con 106 postulantes y Liceo Lastarria con 99 postulantes. Todos ellos corresponden a liceos públicos de excelencia. Si además se restringe la muestra de alumnos no seleccionados a rangos de puntaje más altos, es decir más cercanos al puntaje de corte, se observa que el porcentaje que representan estos cuatro colegios aumenta: considerando los alumnos con un puntaje hasta 100 puntos (1 desviación estándar) bajo el puntaje de corte, el porcentaje alcanza el 23\%, mientras que hasta 50 puntos bajo el puntaje de corte se obtiene un $27 \%$. En definitiva, un cuarto de los alumnos no aceptados con mejor rendimiento ingresan a otros liceos públicos de excelencia, la mayoría de ellos de dependencia municipal ${ }^{5}$.

\section{Datos}

La muestra consiste de datos administrativos con información sobre los postulantes al Instituto Nacional durante los años 2000 y 2002. El total de matriculados en el año 2000 alcanzó un total de 670 nuevos alumnos de entre 3.479 postulantes y en 2002 un total de 675 nuevos alumnos de entre 3.800 postulantes. Al respecto, un factor que emerge al analizar estos datos es la entrega de cupos a estudiantes bajo el puntaje de corte debido a que cuentan con la recomendación de una persona capaz de influir en el proceso de selección. Específicamente, para el año 2000, 94 alumnos tuvieron un puntaje de postulación bajo el puntaje de corte y fueron seleccionados en este proceso especial, lo que corresponde a un $14 \%$ del total de seleccionados. Para el año 2002, el número fue 82 , lo que representó un $12 \%$ del total de seleccionados. Este fenómeno se conoce en el establecimiento como "pituto" y es lo que justifica el uso de la metodología de "regresión discontinua fuzzy" que presentaremos más adelante.

\footnotetext{
${ }^{5}$ Estos datos son presentados en los anexos n ${ }^{\circ} 1,2$ y 3 .
} 
Se dispone además del puntaje obtenido por cada postulante en la Prueba de Admisión (PA) al Instituto Nacional y el puntaje obtenido por los mismos alumnos seis años más tarde, en la Prueba de Selección Universitaria (PSU).

Con objeto de utilizar la información de ambos cohortes (postulantes 2000 y postulantes 2002) de manera conjunta, estandarizamos los puntajes de la PA respecto del puntaje de corte del Instituto Nacional cada año. En particular, cada observación de puntaje estandarizado de la PA corresponde a:

$$
\text { Puntaje Estandarizado } \mathrm{PA}_{i t}=\frac{{\text { Puntaje } \mathrm{PA}_{i t}-\text { Puntaje Corte }_{t}}_{\text {Desviación Estándar }_{t}}}{\text { Dén }}
$$

Donde $i$ indexa al postulante y $t$ indexa a los cohortes 2000 o 2002.

En la tabla $\mathrm{n}^{\circ} 1$ se presenta una estadística descriptiva respecto de los resultados en las PSU de lenguaje y matemáticas para estudiantes seleccionados y no seleccionados de ambos años.

TABLA N ${ }^{\circ}$ 1: $\quad$ ESTADÍSTICA DESCRIPTIVA DE LA PSU, SELECCIONADOS Y NO SELECCIONADOS

\begin{tabular}{cccc}
\hline $\begin{array}{c}\text { Pruebas de } \\
\text { Selección } \\
\text { Universitaria } \\
\text { (PSU) }\end{array}$ & $\begin{array}{c}\text { Seleccionados } \\
(1.240 \text { observaciones })\end{array}$ & $\begin{array}{c}\text { No seleccionados } \\
(4.837 \text { observaciones })\end{array}$ & $\begin{array}{c}\text { Test de medias } \\
(6.076 \text { observaciones })\end{array}$ \\
\hline Puntaje PSU & 673,99 & 567,73 & $-106,3^{* * *}$ \\
Lenguaje & $(66,34)$ & $(87,15)$ & $(-40,06)$ \\
Puntaje PSU & 684,17 & 579,32 & $-104,8 * * *$ \\
Matemáticas & $(74,48)$ & $(88,01)$ & $(-38,55)$ \\
\hline
\end{tabular}

Seleccionados y No seleccionados: Desviación estándar en paréntesis. Test de medias: estadístico $t$ entre paréntesis.

***: Significancia estadística al $1 \%$.

Fuente: Construcción propia en base a datos administrativos del Instituto Nacional.

De la tabla $n^{\circ} 1$, destaca la estrecha relación entre mejores resultados en la PSU y haber sido seleccionado por el Instituto Nacional. De hecho, los puntajes en la PSU de los alumnos seleccionados son estadísticamente mayores a los puntajes de los no seleccionados y poseen menor desviación estándar. 
FIGURA N ${ }^{\circ}$ 2: RELACIÓN ENTRE EL PUNTAJE EN LA PSU Y EL PUNTAJE EN LA PRUEBA DE ADMISIÓN DEL INSTITUTO NACIONAL

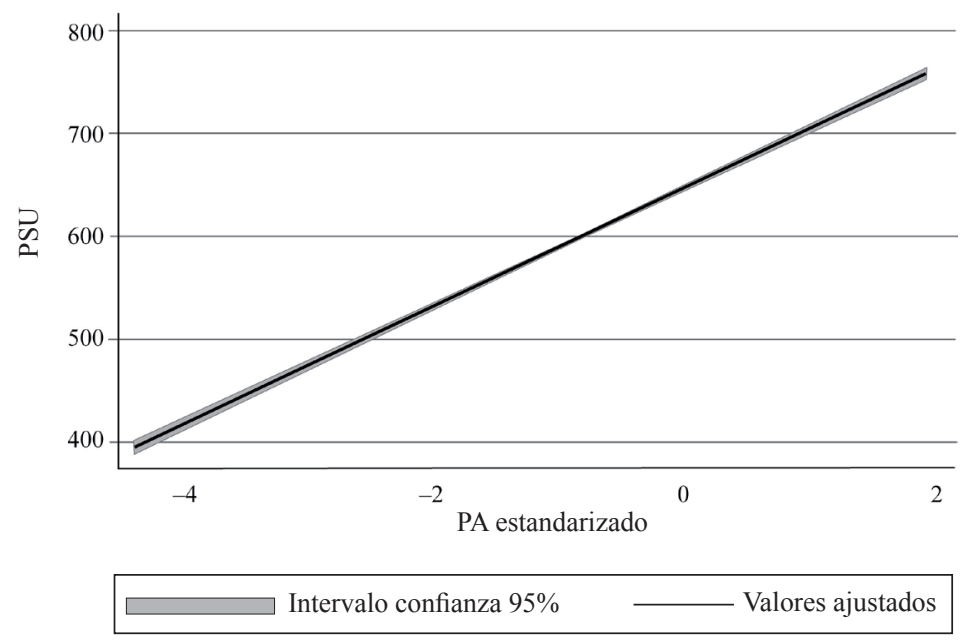

Fuente: Construcción propia en base a datos administrativos del Instituto Nacional.

Por otro lado, el resultado en la prueba de admisión del Instituto Nacional parece estar estrechamente ligada al puntaje obtenido en la PSU. La figura $n^{\circ} 2$ muestra esta relación, lo que sugiere que el puntaje de admisión captura habilidades similares a las medidas en la PSU.

Nuestros resultados demuestran que la correlación entre el puntaje en la PA (estandarizado) y el puntaje en la PSU es de 0,6636 y el R cuadrado de la regresión presentada en la figura $n^{\circ} 2$ es de 0,4403 . En la siguiente sección utilizamos esta lógica para estimar el efecto causal local de asistir al Instituto Nacional.

\section{Metodología}

El diseño de regresión discontinua (RD) pretende identificar relaciones de causalidad locales. La idea principal tras este diseño ${ }^{6}$ es explotar la discontinuidad observable en la probabilidad de tratamiento

6 Siguiendo a Lee y Lemieux (2010), resulta más fácil entender el diseño de la RD como una descripción particular del proceso generador de datos que como un método o enfoque. 
en relación con una variable de asignación $Z$. Es decir, para implementar RD se requiere que la probabilidad de tratamiento tenga un cambio discontinuo en algún valor de $Z$, digamos $Z 0$. En general, bajo supuestos plausibles, se puede señalar que en la vecindad de $Z 0$ un cambio discontinuo en la variable de interés $(Y)$ se puede atribuir al cambio en la probabilidad de tratamiento. Además, en torno a $Z 0$ el nivel de tratamiento puede ser considerado una asignación aleatoria, como si se tratase de un diseño experimental. De lo anterior se desprende que los parámetros estimados por el método de RD posean la mayor validez interna entre los estimadores cuasi experimentales (Lee, 2008).

Imbens y Lemieux (2008) y Lee y Lemieux (2010) discuten los aspectos prácticos y teóricos asociados a la implementación de la RD. Allí se distingue entre dos diseños: regresión discontinua sharp (RDS) y regresión discontinua fuzzy (RDF). En el caso de RDS se tiene que la probabilidad de tratamiento es una función determinística de la variable de asignación $Z$, es decir, en el diseño RDS la probabilidad de tratamiento pasa de cero a uno en $Z 0$. Esto es, todas las observaciones cuyo valor $Z$ sea mayor o igual a $Z 0$ forman parte del grupo de tratamiento, mientras que todas las observaciones cuyo valor $Z$ sea menor a $Z 0$ forman parte del grupo control. Por su parte, en el caso de RDF la probabilidad de tratamiento no es una función determinística de la variable de asignación $Z$, es decir, la probabilidad de tratamiento aumenta discontinuamente en $Z 0$ pero sin cambiar de manera discreta de cero a uno. En este caso, el estimador local del efecto causal, símil del estimador local obtenido con variables instrumentales ${ }^{7}$, corresponde al cambio en $Y$ dividido por el cambio en la probabilidad de ser tratado en $Z 0$.

En estricto rigor, para inferir un efecto causal en $Y$ debido a la discontinuidad en la probabilidad de ser tratado, en nuestro caso de ser aceptado en el Instituto Nacional, en Z0 (puntaje de corte en la prueba de postulación) se requiere de los siguientes supuestos: i) el cambio en $Z 0$ de la probabilidad de ser tratado debe ser discontinuo ${ }^{8}$, ii) $Z$ debe ser

${ }^{7}$ El enfoque de RD es el de un modelo de variables instrumentales (VI) con la variable de asignación $Z$ como variable exógena excluida de la regresión y una variable de tratamiento como un regresor endógeno. Esta relación entre RDF y VI ha sido considerada en Angrist y Krueger (1991) e Imbens y Van Der Klaauw (1995), entre otros.

${ }^{8}$ Este supuesto es de particular importancia en un diseño RDF, tan importante como lo es verificar la existencia de una relación fuerte en la primera etapa de un diseño por VI. 
observado sin error, iii) $Y$ debe ser una función continua de $Z$ en $Z 0$ para todos aquellos individuos no tratados y iv) los individuos no pueden estar ordenados en torno a $Z 0$ de acuerdo a su capacidad de responder al tratamiento. Ahora bien, una de las principales ventajas del diseño de regresión discontinua es su transparencia, la que se puede ilustrar mediante el uso de métodos gráficos, cuestión a desarrollar en la siguiente sección de Resultados y robustez.

\section{Resultados y robustez}

Para esta sección, considérese la siguiente notación: denotamos por $X T$ el nivel de tratamiento, es decir, la probabilidad de ser admitido en el Instituto Nacional; $Z$ es la variable de asignación, es decir, el puntaje estandarizado en la PA del establecimiento, e $Y$ es nuestra

FIGURA N ${ }^{\circ}$ 3: $\quad$ PROBABILIDAD DE SER SELECCIONADO COMO FUNCIÓN DEL PUNTAJE EN LA PRUEBA DE ADMISIÓN

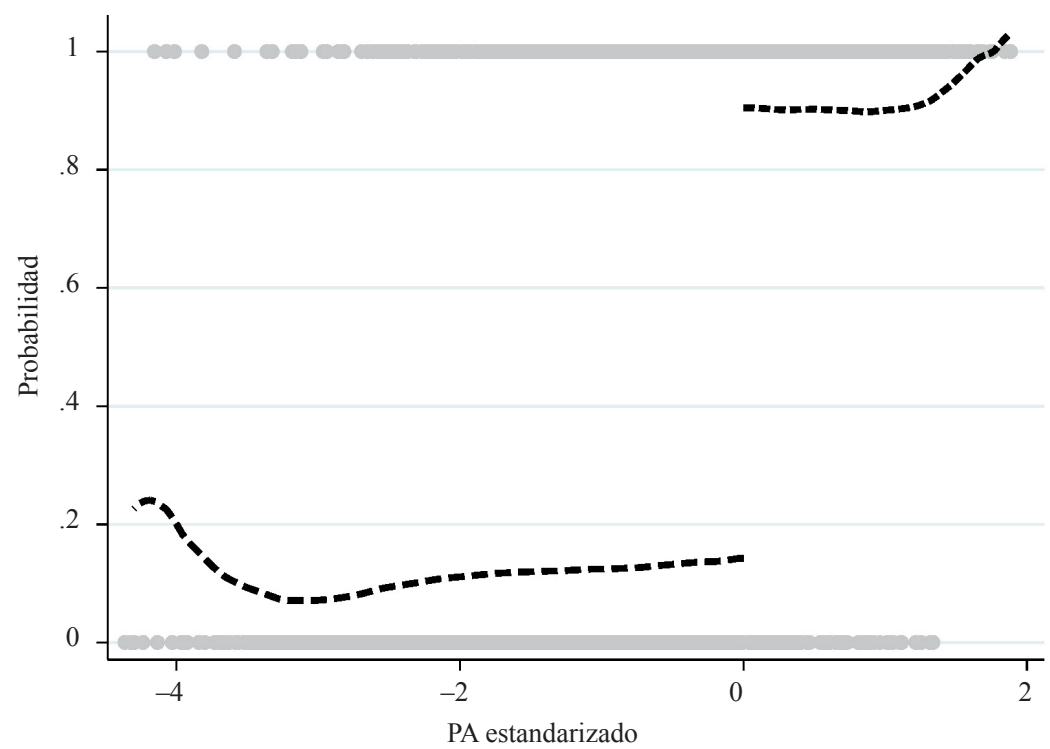

Fuente: Construcción propia en base al programa de Nichols (2007). 
variable de interés o el puntaje en la PSU (promedio simple de los puntajes en lenguaje y matemáticas). Se debe notar que la probabilidad de ser seleccionado por el Instituto Nacional no es una función uno a uno del puntaje obtenido en la PA, es decir, a pesar de que la probabilidad de tratamiento aumenta discontinuamente en torno al punto de corte, este cambio no es discreto (de cero a uno) como sugiere el diseño de la RD sharp. Esto se debe a dos factores, a saber: i) existen alumnos que pueden ser seleccionados "por pituto" y ii) existen alumnos que siendo seleccionados deciden no asistir al Instituto Nacional. La figura $\mathrm{n}^{\circ} 3$ ilustra esta situación.

Dado que la probabilidad de tratamiento no es una función determinística de la variable de asignación, lo pertinente es emplear un diseño de RD fuzzy para estimar el efecto de asistir al Instituto Nacional. El resultado gráfico de la estimación obtenida por esta estrategia se presenta en la figura $n^{\circ} 4$.

FIGURA N ${ }^{\circ}$ 4: $\quad$ REGRESIÓN DISCONTINUA FUZZY

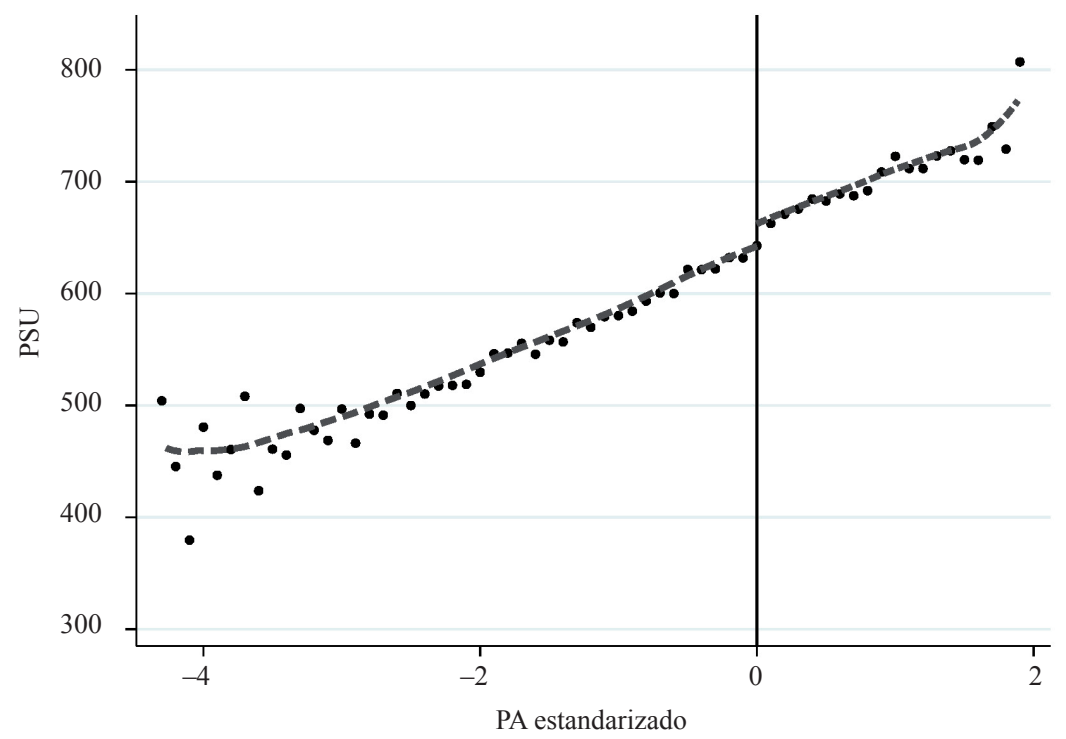

Fuente: Construcción propia en base a datos administrativos del IN. Para efectos de la estimación de RD fuzzy se utilizó un ancho de banda óptimo (Imbens y Kalyanaraman, 2010) el que se presenta en la figura $n^{\circ} 4$. 
En la figura $\mathrm{n}^{\circ} 4$ es evidente la discontinuidad en PSU generada por el evento "asistir al Instituto Nacional". El efecto estimado es de 26,13 puntos adicionales en el puntaje de la PSU ( 0,25 desviaciones estándar). Este efecto es positivo y estadísticamente significativo para distintos anchos de banda, tal como se presenta a continuación en la tabla $\mathrm{n}^{\circ} 2$.

TABLA N ${ }^{\circ}$ 2: $\quad$ REGRESIÓN DISCONTINUA FUZZY:

ESTIMACIONES PARA DISTINTOS ANCHOS DE BANDA ${ }^{9}$

\begin{tabular}{ccc}
\hline \multicolumn{3}{c}{ Efecto del tratamiento sobre el puntaje PSU } \\
\hline Ancho de banda & Coeficiente & Error estándar \\
\hline 0,88 & 26,13 & $(5,95)$ \\
0,44 & 23,29 & $(7,91)$ \\
1,76 & 24,27 & $(4,6)$ \\
\hline Observaciones & \multicolumn{3}{c}{6.076} \\
\hline
\end{tabular}

Fuente: Construcción propia en base a datos administrativos del Instituto Nacional.

Para poner nuestro resultado en contexto, los 26.13 puntos adicionales en PSU equivalen al 95\% de la brecha entre los establecimientos públicos y particulares subvencionados en PSU (datos 2005).

\section{Efecto del pituto}

Adicionalmente, con el cohorte del año 2002 es posible comparar las medias en las pruebas de Sistema de Medición de los Resultados de Aprendizaje de los años 2004 (SIMCE 2004) y 2006 (SIMCE 2006) de todos aquellos estudiantes que fueron seleccionados por el Instituto Nacional a través de "pituto" con aquellos que no fueron admitidos pese a tener un puntaje de admisión similar. Lo anterior nos permite complementar la evidencia antes presentada mediante el método de $\mathrm{RD}$, el que por su naturaleza informa sobre el efecto local de asistir al IN entre quienes están en la vecindad del puntaje de corte. Los alumnos seleccionados por "pituto" nos permiten mostrar el efecto de asistir al Instituto Nacional para aquellos que no están en la vecindad del puntaje de corte. $n^{\circ} 4$.

${ }^{9}$ Los gráficos para distintos anchos de banda se presentan en el Anexo 
Para desarrollar este ejercicio se compara primero a los alumnos seleccionados por "pituto" con un puntaje de admisión de hasta 50 puntos bajo el puntaje de corte versus alumnos no seleccionados con el mismo puntaje de admisión. Los resultados muestran que existen diferencias a favor de los alumnos seleccionados "por pituto" en las pruebas de matemáticas del SIMCE y PSU, además de la prueba de lenguaje de la PSU. Las diferencias en la PSU corresponden a 38 y 30 puntos respectivamente, siendo ambas estadísticamente significativas. Ahora bien, los alumnos no seleccionados por el Instituto Nacional pero con un puntaje similar al de aquellos seleccionados "por pituto" muestran una diferencia a su favor y significativa en el puntaje asociado a las notas de enseñanza media (NEM).

Esta ventaja a favor de los alumnos que asistieron al Instituto Nacional pese a estar bajo el puntaje de corte se mantiene al alejarnos de este corte en 100 e incluso al considerar a todos los alumnos que asistieron al Instituto Nacional pese a estar bajo el puntaje de corte ${ }^{10}$.

\section{Prueba de McCrary}

Un elemento clave que valida la estrategia de identificación basada en la RD es la verificación de que la variable de asignación sea continua en torno al punto de corte. Siguiendo a McCrary (2008), en la figura $\mathrm{n}^{\circ} 5$ se muestra la densidad de dicha variable, con un intervalo de confianza del $95 \%$. Es evidente notar que en torno al puntaje de corte $(Z O=0)$ el intervalo de confianza a ambos lados se intersecta y, por tanto, no es posible rechazar la hipótesis de continuidad de la variable de asignación en torno al puntaje de corte establecido cada año por el IN.

En base a este resultado, podemos concluir que el diseño utilizado en este estudio es pertinente para evaluar el efecto del Instituto Nacional sobre los puntajes en la PSU.

\section{Ejercicios de robustez}

Para efectos de asegurar la robustez de nuestros resultados, en esta sección presentamos distintos ejercicios de robustez.

En primer lugar, analizamos la sensibilidad de nuestros resultados a distintos anchos de banda. La figura $n^{\circ} 6$ presenta los efectos estimados por RD fuzzy, con intervalos al 95\% de confianza, para doce

\footnotetext{
${ }^{10}$ Estos resultados son presentados en los Anexos n ${ }^{\circ} 5,6$ y 7 .
} 
FIGURA N ${ }^{\circ}$ 5: $\quad$ DENSIDAD DEL PUNTAJE EN LA PRUEBA DE ADMISIÓN. CONTINUIDAD EN TORNO AL PUNTO DE CORTE

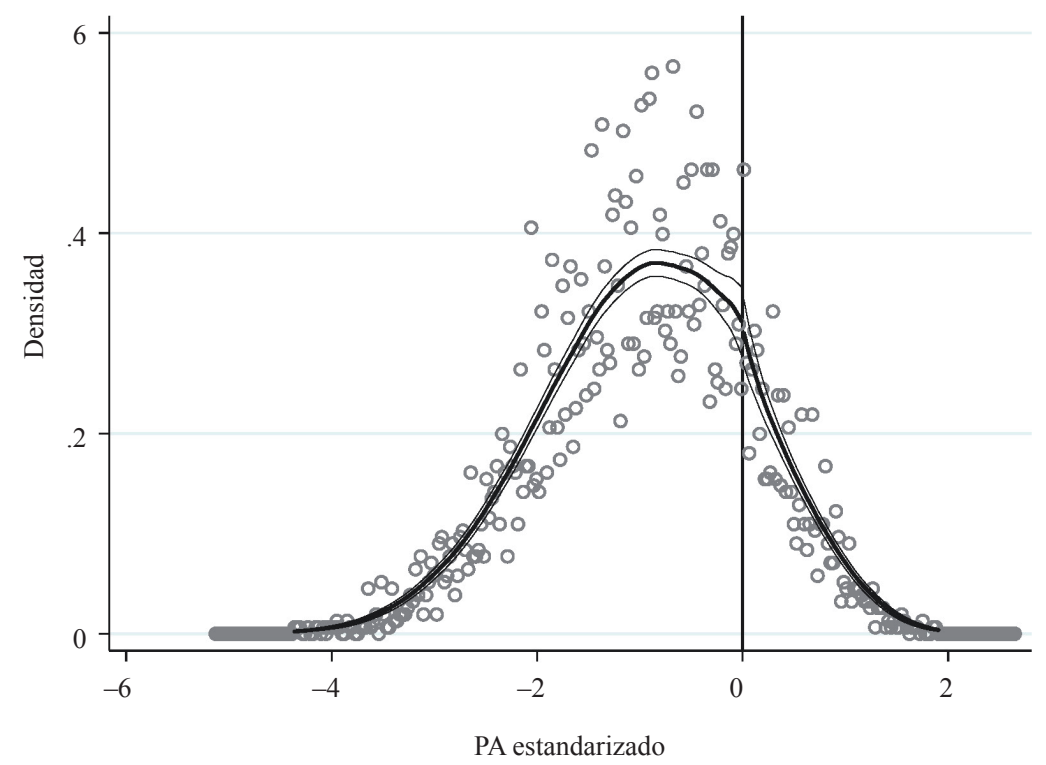

Fuente: Construcción propia en base a datos administrativos del IN y al programa de McCrary (2008).

anchos de banda distintos. A partir de los resultados, se puede concluir que el efecto de asistir al Instituto Nacional es positivo y estadísticamente significativo en un $92 \%$ de las estimaciones realizadas con el diseño de RD fuzzy, lo que demuestra que la selección del ancho de banda no es un elemento importante en nuestras conclusiones.

Un segundo factor que consideramos es el impacto de acotar la muestra en torno al puntaje de corte. En particular, la tabla $n^{\circ} 3$ presenta el efecto estimado del tratamiento para distintos anchos de banda, considerando una submuestra de estudiantes con puntajes ubicados 1 y 0,5 desviaciones estándar del puntaje de corte en la PA del Instituto Nacional $^{11}$.

${ }^{11}$ En el Anexo $n^{\circ} 8$ se presentan también los resultados para la submuestra de estudiantes ubicados a 0,25 desviaciones estándar del puntaje de corte de la prueba de admisión. 
FIGURA N ${ }^{\circ}$ 6: REGRESIÓN DISCONTINUA FUZZY: ESTIMACIONES PARA DISTINTOS ANCHOS DE BANDA

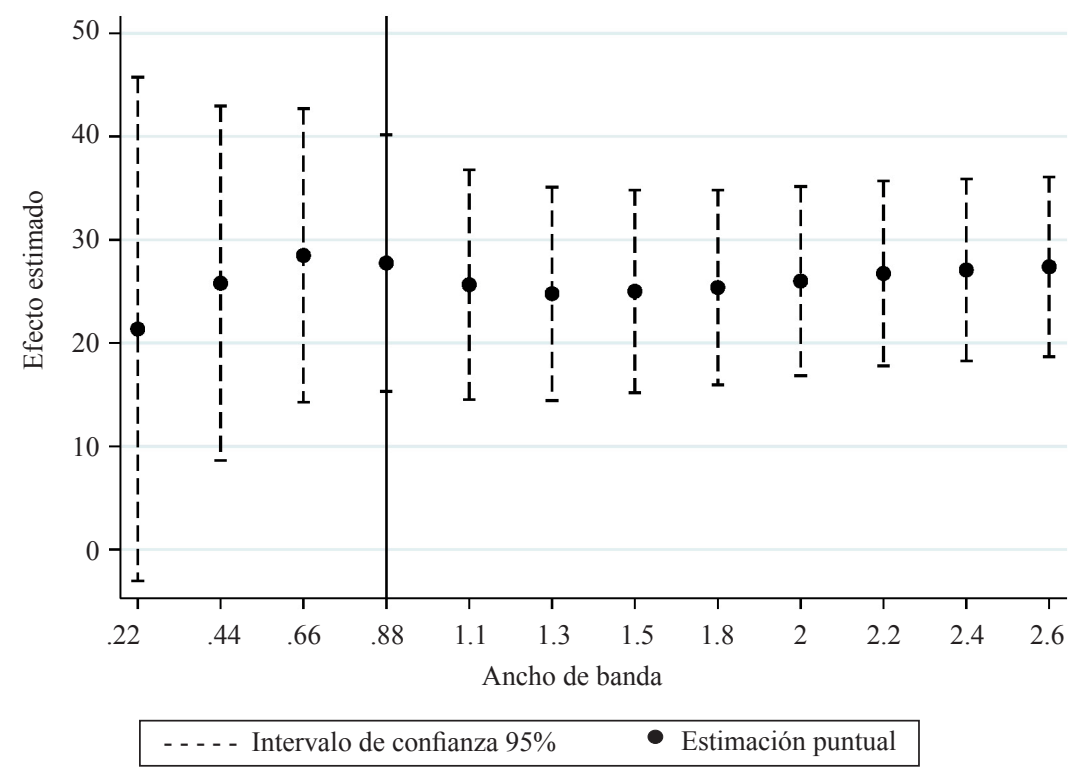

Fuente: Construcción propia en base al programa de Nichols (2007).

TABLA N ${ }^{\circ}$ 3: $\quad$ EFECTO DEL TRATAMIENTO

\begin{tabular}{cccccc}
\hline $\begin{array}{c}\text { Efecto del tratamiento sobre el puntaje PSU } \\
\text { (muestra acotada 1 SD) }\end{array}$ & \multicolumn{3}{c}{$\begin{array}{c}\text { Efecto del tratamiento sobre el puntaje PSU } \\
\text { (muestra acotada 0,5 SD) }\end{array}$} \\
\hline $\begin{array}{c}\text { Ancho de } \\
\text { banda }\end{array}$ & Coeficiente Error estándar & $\begin{array}{c}\text { Ancho de } \\
\text { banda }\end{array}$ & Coeficiente & Error estándar \\
\hline 0,71 & 26,45 & $(6,50)$ & 0,21 & 20,55 & $(12,33)$ \\
0,35 & 23,5 & $(9,02)$ & 0,10 & 24,61 & $(15,84)$ \\
1,42 & 23,52 & $(5,33)$ & 0,41 & 23,35 & $(8,28)$ \\
\hline Observaciones & 3.179 & Observaciones & \multicolumn{3}{c}{1.712} \\
\hline
\end{tabular}

Fuente: Construcción propia en base a datos administrativos del Instituto Nacional.

A partir de la tabla $n^{\circ} 3$, podemos concluir que nuestros resultados son robustos en signo y magnitud a este ejercicio. El aumento en los errores estándar se explica por la significativa reducción en el tamaño muestral. 
Podemos también utilizar nuestros datos para realizar una prueba de falsificación. En particular, podemos arbitrariamente modificar el punto de corte de la PA en $-0,2$ y $+0,2$ desviaciones estándar. Es de esperar que un desplazamiento en el punto de corte genere estimaciones no estadísticamente significativas. Las estimaciones que se presentan en la tabla $\mathrm{n}^{\circ} 4$ confirman la pertinencia de nuestro enfoque.

TABLA N ${ }^{\circ}$ 4: $\quad$ EFECTO DEL TRATAMIENTO CONSIDERANDO DESPLAZAMIENTOS EN EL PUNTO DE CORTE

\begin{tabular}{cccccc}
\hline \multicolumn{2}{c}{ (Punto de corte + 0,2 desv. est.) } & \multicolumn{3}{c}{ (Punto de corte - 0,2 desv. est.) } \\
\hline $\begin{array}{c}\text { Ancho de } \\
\text { banda }\end{array}$ & Coeficiente & Error estándar & $\begin{array}{c}\text { Ancho de } \\
\text { banda }\end{array}$ & Coeficiente & Error estándar \\
\hline 0,64 & 46,82 & $(48,36)$ & 1,21 & 29,87 & $(23,20)$ \\
0,32 & $-62,96$ & $(67,46)$ & 0,61 & $-4,68$ & $(15,95)$ \\
1,28 & 7,92 & $(29,51)$ & 2,42 & 26,53 & $(8,49)$ \\
\hline Observaciones & & & 6.076 & \\
\hline
\end{tabular}

Fuente: Construcción propia en base a datos administrativos del Instituto Nacional.

Finalmente, un posible problema con nuestra estrategia empírica sería que quienes fueron seleccionados para ingresar al Instituto Nacional difieran en observables respecto de quienes en el margen no fueron aceptados. Para comparar ambos grupos empleamos una característica que en la literatura de economía de la educación es considerada determinante de los resultados académicos de los estudiantes: educación de los padres. La tabla $n^{\circ} 5$ presenta la estimación por el método de $\mathrm{RD}$ del efecto del tratamiento en la probabilidad de que la madre del estudiante tenga al menos educación media completa ${ }^{12}$. Como vemos, para varios anchos de banda, quienes están por debajo del puntaje de corte no difieren de manera significativa de quienes están por sobre éste.

Este ejercicio muestra que los estudiantes cuyos puntajes se ubican en torno al puntaje de corte de la prueba de admisión son similares entre sí y, por tanto, nuestras estimaciones capturan el efecto de asistir al Instituto Nacional para dicho grupo.

${ }^{12}$ Resultados similares se encuentran al usar educación del padre. 
TABLA N ${ }^{\circ}$ 5: $\quad$ EFECTO DEL TRATAMIENTO SOBRE LA PROBABILIDAD DE QUE LA MADRE TENGA AL MENOS EDUCACIÓN MEDIA. POSTULANTES AL INSTITUTO NACIONAL AÑO 2002

\begin{tabular}{ccc}
\hline Ancho de banda & Coeficiente & Error estándar \\
\hline 0,87 & -0.04 & $(0,06)$ \\
0,43 & $-0,001$ & $(0,08)$ \\
1,73 & $-0,03$ & $(0,05)$ \\
\hline
\end{tabular}

Observaciones

2.966

Fuente: Construcción propia en base a datos administrativos del Instituto Nacional.

\section{Conclusión}

En este trabajo se ha presentado evidencia del efecto de asistir al Instituto Nacional sobre los resultados académicos de los jóvenes. En particular, encontramos que el efecto de asistir a este liceo público de excelencia es de 26,13 puntos adicionales en la PSU, esto es: 0,25 desviaciones estándar. Este efecto corresponde a un LATE (Local Average Treatment Effect) y es robusto frente a la estimación con distintos anchos de banda. En este sentido, se puede señalar que los esfuerzos de las familias y de los estudiantes (proceso de postulación, largos tiempos de viaje, etcétera) por acceder a este establecimiento se ven recompensados. Nuestros resultados sugieren que el Instituto Nacional tiene efectos importantes en la prueba de selección universitaria para los alumnos que se encuentran próximos al puntaje de corte.

La evidencia aquí presentada puede ser una contribución a la discusión respecto de la implementación de liceos públicos de excelencia. El tema es relevante toda vez que este tipo de liceos pudiese permitir aumentar la escasa movilidad social existente en Chile, contribuyendo con ello a una mayor igualdad de oportunidades. Ahora bien, se debe reconocer que la presente investigación no aborda el canal por el cual el Instituto Nacional genera mejores resultados, en particular no es posible señalar si el mayor puntaje en la PSU se debe a efecto par, tracking u otros aspectos. Este tema pudiese ser objeto de futuras investigaciones. Por último, también reconocemos que, por sus características, no es directamente posible extrapolar nuestros estimadores locales a otros establecimientos educacionales. Su interpretación como impactos globales del Instituto Nacional también estaría sujeta a cualificaciones. 


\section{Anexos}

Anexo $\mathrm{n}^{\circ}$ 1: Alumnos no aceptados 200 (sin restricción de puntaje)

\begin{tabular}{lrccc}
\hline Nombre establecimiento & RBD & $\begin{array}{c}\mathrm{N}^{\circ} \text { alum- } \\
\text { nos no } \\
\text { aceptados }\end{array}$ & $\begin{array}{c}\text { Porcen- } \\
\text { taje de la } \\
\text { muestra }\end{array}$ & Dependencia \\
\hline Liceo Nacional de Maipú & 25.770 & 205 & 7,1 & Municipal \\
Liceo de Aplicación & 8.491 & 186 & 6,4 & Municipal \\
Internado Nacional Barros Arana & 8.499 & 106 & 3,7 & Municipal \\
Liceo José Victorino Lastarria & 8.928 & 99 & 3,4 & Municipal \\
Liceo Andrés Bello & 9.406 & 40 & 1,4 & Municipal \\
Colegio Piamarta & 24.685 & 32 & 1,1 & Subvencionado \\
Escuela Salvador Sanfuentes & 8.552 & 27 & 0,9 & Municipal \\
Escuela Básica Reyes Católicos & 8.756 & 23 & 0,8 & Municipal \\
Escuela Particular Francisco Andrés Olea & 8.659 & 20 & 0,7 & Subvencionado \\
Instituto La Salle & 9.317 & 20 & 0,7 & Subvencionado \\
\hline
\end{tabular}

\section{Anexo $\mathrm{n}^{\circ}$ 2: Alumnos no aceptados 2002 (hasta 100 puntos bajo puntaje de corte)}

\begin{tabular}{lcccc}
\hline Nombre establecimiento & RBD & $\begin{array}{c}\mathrm{N}^{\circ} \text { alum- } \\
\text { nos no } \\
\text { aceptados }\end{array}$ & $\begin{array}{c}\text { Porcen- } \\
\text { taje de la } \\
\text { muestra }\end{array}$ & Dependencia \\
\hline Liceo Nacional de Maipú & 25.770 & 190 & 8,2 & Municipal \\
Liceo de Aplicación & 8.491 & 167 & 7,2 & Municipal \\
Liceo José Victorino Lastarria & 8.499 & 94 & 4,1 & Municipal \\
Internado Nacional Barros Arana & 8.928 & 94 & 4,1 & Municipal \\
Liceo Andrés Bello & 9.406 & 38 & 1,6 & Municipal \\
Escuela Salvador Sanfuentes & 8.552 & 27 & 1,2 & Municipal \\
Colegio Piamarta & 24.685 & 25 & 1,1 & Subvencionado \\
Escuela Básica Reyes Católicos & 8.756 & 19 & 0,8 & Municipal \\
Escuela Particular Francisco Andrés Olea & 8.659 & 18 & 0,8 & Subvencionado \\
Instituto La Salle & 9.317 & 18 & 0,8 & Subvencionado \\
\hline
\end{tabular}


Anexo $\mathrm{n}^{\circ}$ 3: Alumnos no aceptados 2002

(hasta 50 puntos bajo puntaje de corte)

\begin{tabular}{lcccc}
\hline Nombre establecimiento & RBD & $\begin{array}{c}\mathrm{N}^{\circ} \text { alum- } \\
\text { nos no } \\
\text { aceptados }\end{array}$ & $\begin{array}{c}\text { Porcen- } \\
\text { taje de la } \\
\text { muestra }\end{array}$ & Dependencia \\
\hline Liceo Nacional de Maipú & 25.770 & 112 & 8,94 & Municipal \\
Liceo de Aplicación & 8.491 & 107 & 8,54 & Municipal \\
Liceo José Victorino Lastarria & 8.928 & 69 & 5,51 & Municipal \\
Internado Nacional Barros Arana & 8.499 & 60 & 4,79 & Municipal \\
Liceo Andrés Bello & $9 . .406$ & 21 & 1,68 & Municipal \\
Escuela Salvador Sanfuentes & 8552 & 13 & 1,04 & Municipal \\
Escuela Particular Francisco Andrés Olea & 8.659 & 12 & 0,96 & Subvencionado \\
Colegio Piamarta & 24.685 & 12 & 0,96 & Subvencionado \\
Instituto La Salle & 9.317 & 10 & 0,8 & Subvencionado \\
Escuela Particular y Colegio Chile & 9.504 & 10 & 0,8 & Subvencionado \\
\hline
\end{tabular}


Anexo $n^{\circ} 4$ : Regresión discontinua fuzzy: Ancho de banda 0,44 y 1,75

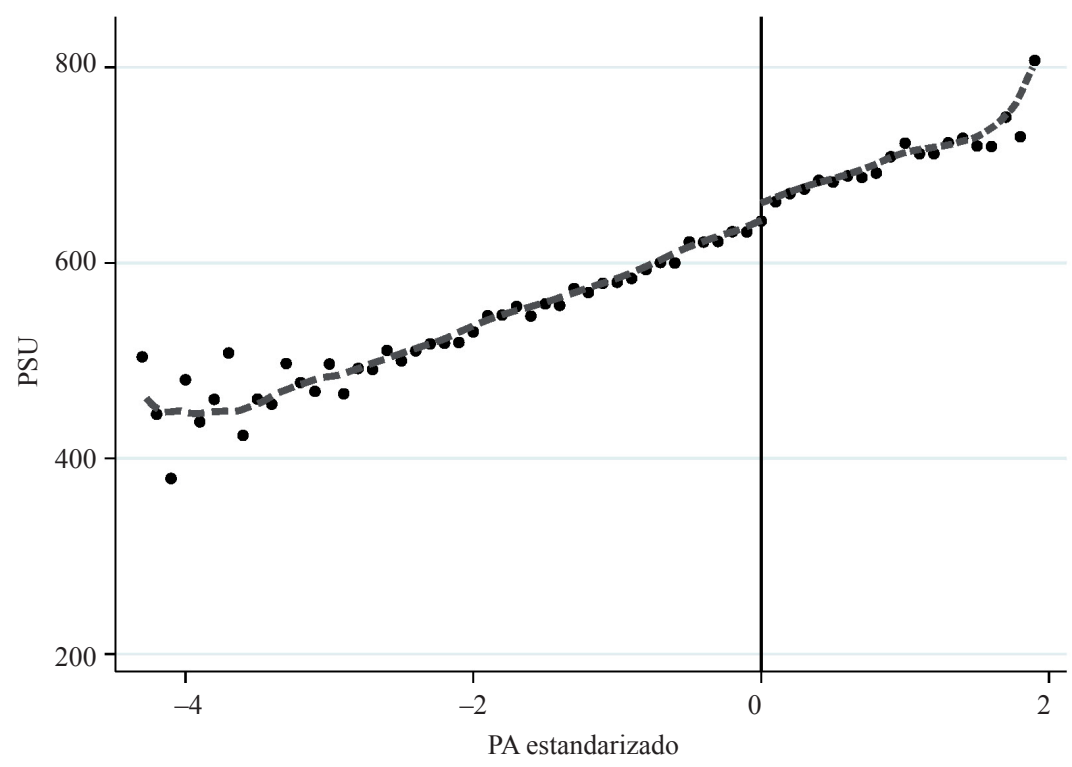

6.076 observaciones. Ancho de banda 0,438. Estimador local de Wald 24,856.

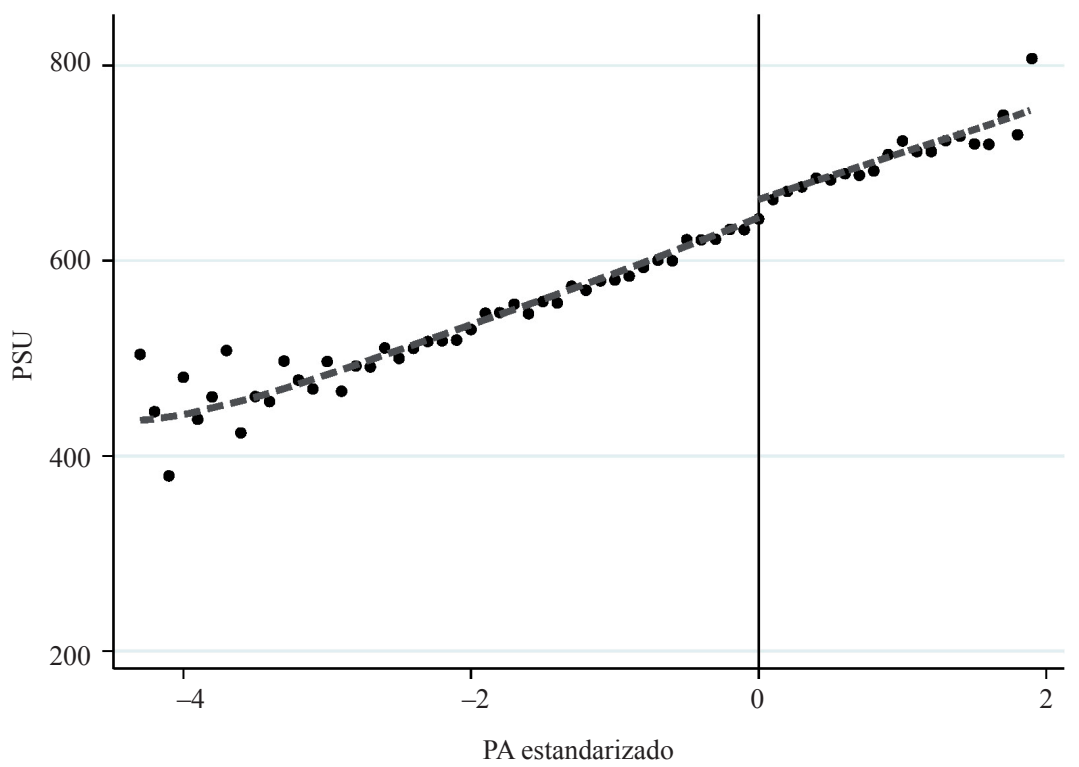

6.076 observaciones. Ancho de banda 1,751. Estimador local de Wald 24,955. 


\section{Anexo $\mathrm{n}^{\circ}$ 5: Comparación de medias pruebas SIMCE y PSU (hasta 50 puntos bajo puntaje de corte)}

\begin{tabular}{|c|c|c|c|c|}
\hline Clasificación del alumno & Leng2004 & Mate2004** & Soc2004 & Nat2004 \\
\hline $\begin{array}{l}\text { Promedio puntaje seleccionados } \\
\text { proceso especial }\end{array}$ & 312,96 & 331,29 & 313,18 & 325,42 \\
\hline $\begin{array}{l}\text { Número de alumnos seleccionados } \\
\text { proceso especial }\end{array}$ & 56 & 54 & 55 & 56 \\
\hline Promedio puntaje no seleccionados & 308,89 & 323,34 & 316,96 & 320,04 \\
\hline Número de alumnos no seleccionados & 1.239 & 1.244 & 1.244 & 1.240 \\
\hline Promedio puntaje (todos) & 309,07 & 323,67 & 316,80 & 320,28 \\
\hline Número de alumnos (todos) & 1.295 & 1.298 & 1.299 & 1.296 \\
\hline Clasificación del alumno & & Leng2006 & \multicolumn{2}{|c|}{ Mate2006** } \\
\hline $\begin{array}{l}\text { Promedio puntaje seleccionados } \\
\text { proceso especial }\end{array}$ & & 311,20 & \multicolumn{2}{|c|}{356,51} \\
\hline $\begin{array}{l}\text { Número de alumnos seleccionados } \\
\text { proceso especial }\end{array}$ & & 44 & \multicolumn{2}{|r|}{45} \\
\hline Promedio puntaje no seleccionados & & 308,30 & \multicolumn{2}{|c|}{337,06} \\
\hline Número de alumnos no seleccionados & & 1.192 & \multicolumn{2}{|c|}{1.194} \\
\hline Promedio puntaje (todos) & & 308,40 & \multicolumn{2}{|c|}{337,77} \\
\hline Número de alumnos (todos) & & 1.236 & \multicolumn{2}{|c|}{1.239} \\
\hline
\end{tabular}

**: Significancia estadística al 5\%. 


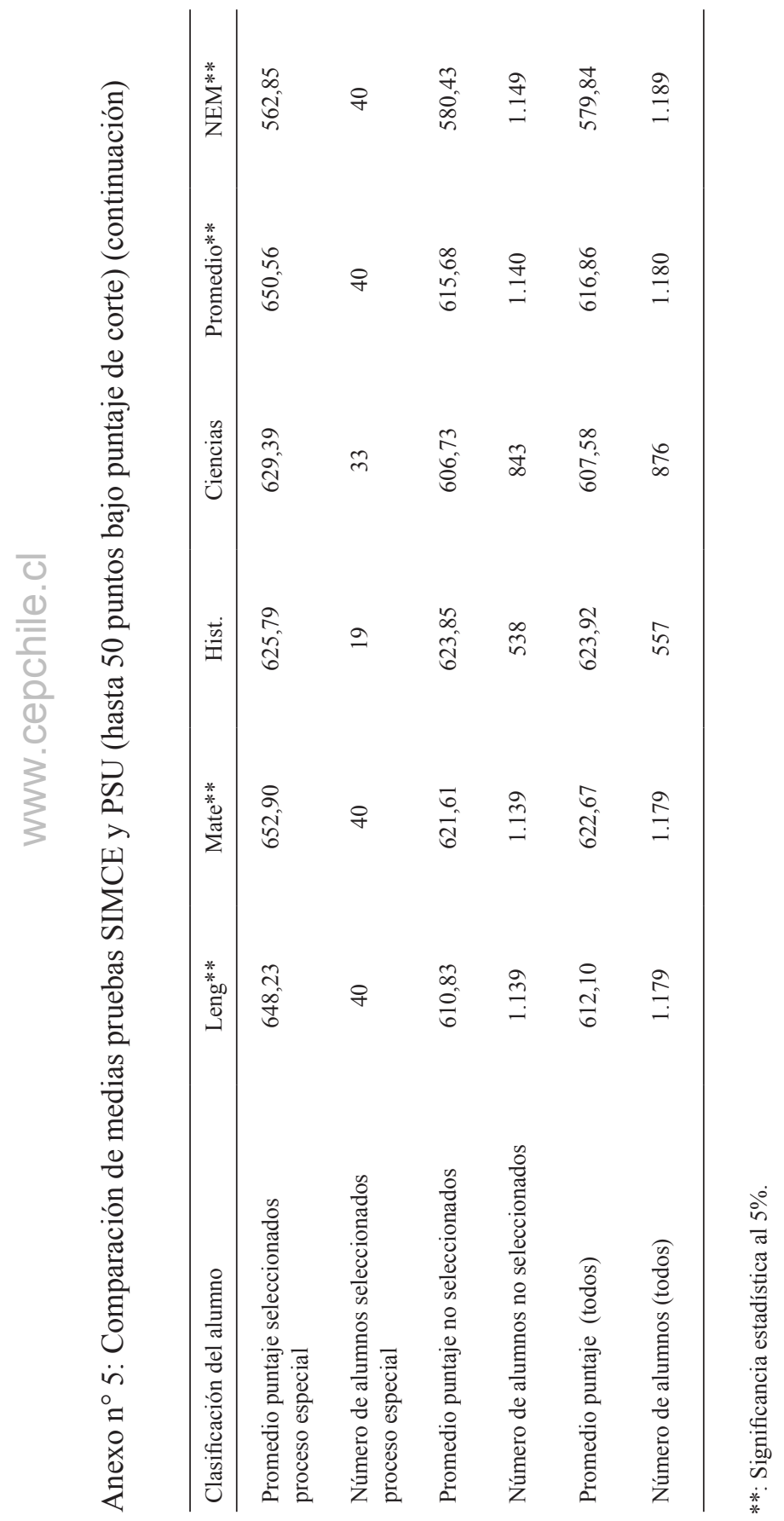




\section{Anexo $n^{\circ}$ 6: Comparación de medias pruebas SIMCE y PSU (hasta 100 puntos bajo puntaje de corte)}

\begin{tabular}{lcccc}
\hline Clasificación del alumno & Leng2004** & Mate2004** & Soc2004 & Nat2004** \\
\hline $\begin{array}{l}\text { Promedio puntaje seleccionados } \\
\text { proceso especial }\end{array}$ & 309,29 & 327,42 & 308,86 & 322,14 \\
$\begin{array}{l}\text { Número de alumnos seleccionados } \\
\text { proceso especial }\end{array}$ & 75 & 73 & 74 & 75 \\
$\begin{array}{l}\text { Promedio puntaje } \\
\text { no seleccionados }\end{array}$ & 299,16 & 312,32 & 307,46 & 309,88 \\
$\begin{array}{l}\text { Número de alumnos } \\
\text { no seleccionados }\end{array}$ & 2.294 & 2.309 & 2.304 & 2.297 \\
$\begin{array}{l}\text { Promedio puntaje (todos) } \\
\text { Número de alumnos (todos) }\end{array}$ & 299,48 & 312,78 & 307,50 & 310,27 \\
\hline
\end{tabular}

\begin{tabular}{lcc}
\hline Clasificación del alumno & Leng2006** & Mate2006** \\
\hline $\begin{array}{l}\text { Promedio puntaje seleccionados } \\
\text { proceso especial }\end{array}$ & 312,15 & 353,51 \\
$\begin{array}{l}\text { Número de alumnos seleccionados } \\
\text { proceso especial }\end{array}$ & 58 & 59 \\
Promedio puntaje no seleccionados & 297,57 & 323,76 \\
Número de alumnos no seleccionados & 2.176 & 2.179 \\
Promedio puntaje (todos) & 297,95 & 324,54 \\
Número de alumnos (todos) & 2.234 & 2.238 \\
\hline
\end{tabular}

\footnotetext{
**: Significancia estadística al 5\%.
} 


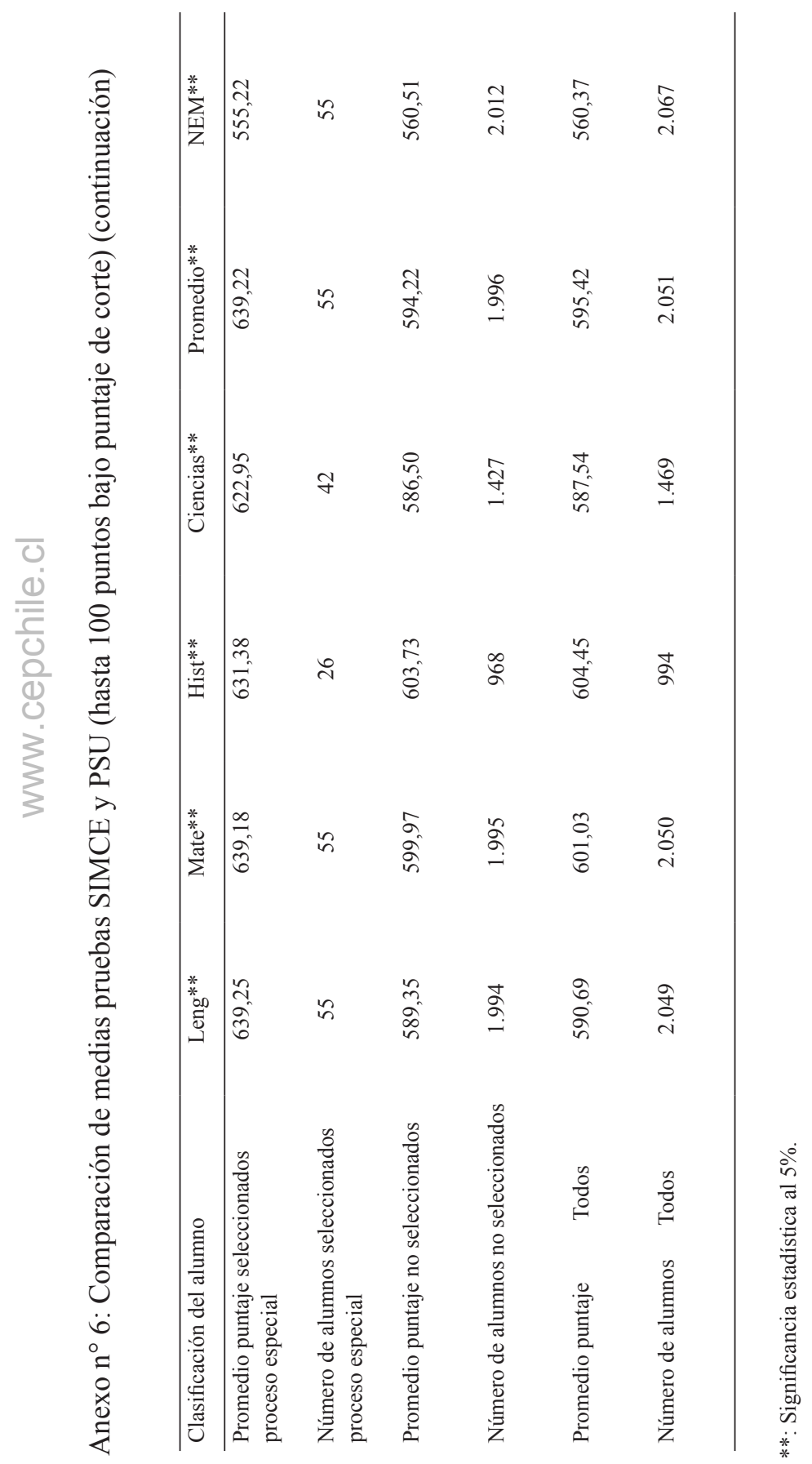




\section{Anexo $n^{\circ} 7$ : Comparación de medias pruebas SIMCE y PSU ( $\sin$ restricción de puntaje)}

\begin{tabular}{|c|c|c|c|c|}
\hline Clasificación del alumno & Leng2004** & Mate2004** & Soc $2004 * *$ & Nat2004** \\
\hline $\begin{array}{l}\text { Promedio puntaje seleccionados } \\
\text { proceso especial }\end{array}$ & 309,01 & 326,40 & 307,95 & 321,63 \\
\hline $\begin{array}{l}\text { Número de alumnos seleccionados } \\
\text { proceso especial }\end{array}$ & 77 & 75 & 76 & 77 \\
\hline $\begin{array}{l}\text { Promedio puntaje } \\
\text { no seleccionados }\end{array}$ & 291,03 & 303,14 & 300,14 & 302,45 \\
\hline $\begin{array}{l}\text { Número de alumnos } \\
\text { no seleccionados }\end{array}$ & 2.953 & 2.979 & 2.972 & 2.957 \\
\hline Promedio puntaje (todos) & 291,49 & 303,71 & 300,33 & 302,93 \\
\hline Número de alumnos (todos) & 3.030 & 3.054 & 3.048 & 3.034 \\
\hline Clasificación del alumno & & Leng2006** & \multicolumn{2}{|c|}{ Mate2006** } \\
\hline $\begin{array}{l}\text { Promedio puntaje seleccionados } \\
\text { proceso especial }\end{array}$ & & 312,10 & \multicolumn{2}{|c|}{352,54} \\
\hline $\begin{array}{l}\text { Número de alumnos seleccionados } \\
\text { proceso especial }\end{array}$ & & 59 & \multicolumn{2}{|r|}{60} \\
\hline Promedio puntaje no seleccionados & & 290,81 & \multicolumn{2}{|c|}{314,20} \\
\hline Número de alumnos no seleccionados & & 2.748 & \multicolumn{2}{|c|}{2.750} \\
\hline Promedio puntaje (todos) & & 291,26 & \multicolumn{2}{|c|}{315,02} \\
\hline Número de alumnos (todos) & & 2.807 & \multicolumn{2}{|c|}{2.810} \\
\hline
\end{tabular}

**: Significancia estadística al 5\%. 


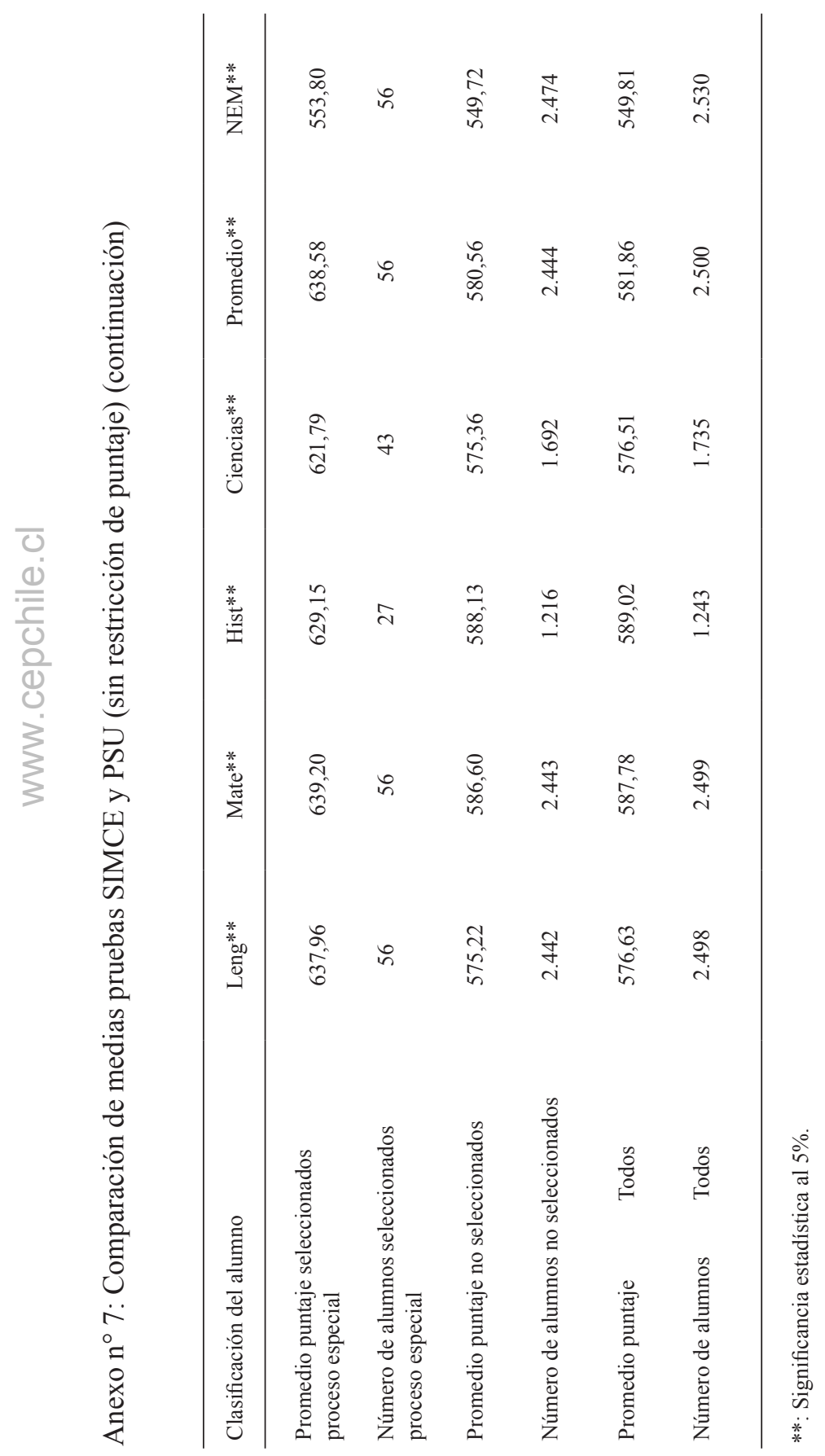


Anexo $\mathrm{n}^{\circ}$ 8: Resultados para la submuestra de estudiantes ubicados a 0,25 desviaciones estándar del puntaje de corte de la prueba de admisión

\begin{tabular}{ccc}
\hline Ancho de banda & Coeficiente & Error estándar \\
\hline 0,16 & 20,02 & 13,22 \\
0,08 & 22,79 & 17,96 \\
0,32 & 21,36 & 10,38 \\
\hline
\end{tabular}

\section{BIBLIOGRAFÍA}

Abdulkadiroğlu, Atila, Joshua Angrist \& Parag A. Pathak (2012). "The Elite Illusion: Achievement Effects at Boston And New York Exam Schools". IZA Discussion Papers 6790, Institute for the Study of Labor.

Allende, Claudio \& Juan Pablo Valenzuela (2012). "Logros en liceos públicos de excelencia en Chile: ¿valor agregado o sólo descreme de la elite?” (mimeo).

Angrist, Joshua D. \& Alan B. Krueger (1991). "Does compulsory school attendance affect schooling and earnings?". Quarterly Journal of Economics 106: 979-1014.

(2001). "Instrumental Variables and the Search for Identification: from Supply and Demand to Natural Experiments". Journal of Economic Perspectives, American Economic Association, 15(4): 69-85.

Clark, Damon (2008). "Selective schools and academic achievement". IZA Discussion papers 3182, Institute for the Study of Labor.

Cullen, Julie Berry, Brian A. Jacob \& Steven Levitt (2006). "The Effect of School Choice on Participants: Evidence from Randomized Lotteries". Econometrica, Econometric Society, 74(5): 1191-1230.

Dobbie, Will \& Roland G. Fryer, Jr. (2011). "Exam High Schools and Academic Achievement: Evidence from New York City". Working Paper 17286, National Bureau of Economic Research.

Duflo, Esther, Pascaline Dupas \& Michael Kremer (2011). "Peer Effects, Teacher Incentives, and the Impact of Tracking: Evidence from a Randomized Evaluation in Kenya". American Economic Review, American Economic Association, 101(5): 1739-74.

Hastings, Justine S. \& Jeffrey M. Weinstein (2008). "Information, School Choice, and Academic Achievement: Evidence from Two Experiments". The Quarterly Journal of Economics, MIT Press, 123(4): 1373-1414.

Imbens, Guido \& Karthik Kalyanaraman (2010). "Optimal Bandwidth Choice for the Regression Discontinuity Estimator". Working paper CWP05/10, Centre for Microdata Methods and Practice, Institute for Fiscal Studies. 
Imbens, Guido \& Thomas Lemieux (2008). "Regression Discontinuity Designs: A Guide to Practice". Journal of Econometrics, Elsevier, 142(2): 615-635.

Imbens, Guido \& Wilbert Van Der Klaauw (1995). "Evaluating the Cost of Conscription in The Netherlands". Journal of Business \& Economic Statistics 13, 72-80.

Jackson, Kirabo (2010). "Do Students Benefit from Attending Better Schools? Evidence from Rule-Based Student Assignments in Trinidad and Tobago". Economic Journal, Royal Economic Society, 120(549): 13991429.

Lee, David S. (2008). "Randomized Experiments from Non-Random Selection in U.S. House Elections." Journal of Econometrics 142(2): 675-697.

Lee, David S. \& Thomas Lemieux (2010). "Regression Discontinuity Designs in Economics". Journal of Economic Literature, American Economic Association, 48(2): 281-355.

McCrary, Justin (2008). "Manipulation of the Running Variable in the Regression Discontinuity Design: A Density Test". Journal of Econometrics, Elsevier, 142(2): 698-714.

Nichols, Austin (2007). "RD: Stata Module for Regression Discontinuity Estimation". Statistical Software Components S456888, Boston College Department of Economics (revised 17 Jun 2012).

Pop-Eleches, Cristian \& Miguel Urquiola (2011). "Going to a Better School: Effects and Behavioral Responses". Working paper 16886, National Bureau of Economic Research.

Sacerdote, Bruce (2011). "Peer Effects in Education: How Might They Work, How Big Are They and How Much Do We Know thus Far?". En Erik Hanushek, Stephen Machin \& Ludger Woessmann (eds.), Handbook of the Economics of Education vol 3, Elsevier, 249-277

Zhang, Hongliang (2010). "Magnet Schools and Student Achievement: Evidence from a Randomized Natural Experiment in China". Job Market Paper, Department of Economics, MIT. 